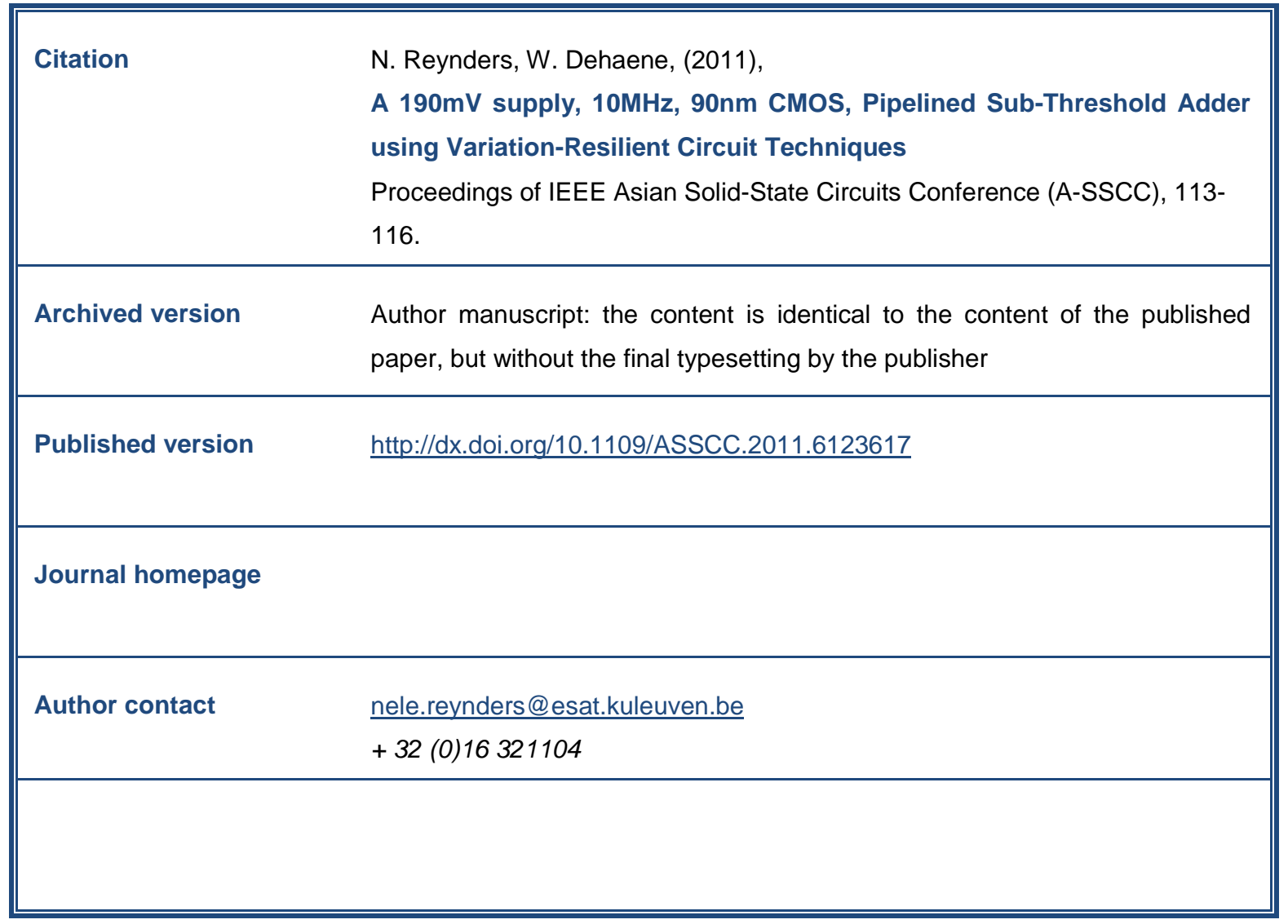

(article begins on next page) 


\title{
A 190mV supply, 10MHz, 90nm CMOS, Pipelined Sub-Threshold Adder using Variation-Resilient Circuit Techniques
}

\author{
Nele Reynders and Wim Dehaene \\ Katholieke Universiteit Leuven, ESAT-MICAS \\ Kasteelpark Arenberg 10, B-3001 Heverlee, Belgium \\ Email: nele.reynders@esat.kuleuven.be
}

\begin{abstract}
This paper presents a pipelined 32 bit sub-threshold adder in a $90 \mathrm{~nm}$ CMOS technology that combines $\mathrm{MHz}$ performance with sub-pJ energy consumption. To increase variation-resilience various circuit techniques are proposed, such as sub-threshold adapted transmission gate logic, optimal sizing for noise margins and time borrowing. These techniques enable operation down to a supply of $190 \mathrm{mV}$ at $10 \mathrm{MHz}$ and an energy consumption of $0.4 \mathrm{pJ}$ per addition. A performance of $30 \mathrm{MHz}$ is obtained at a supply of $260 \mathrm{mV}$ and $0.6 \mathrm{pJ}$ per addition. The adder achieves an improvement in Energy-Delay Product of a factor 900 compared to the state-of-the-art sub-threshold adder design.
\end{abstract}

\section{INTRODUCTION}

Ultra-low-power digital circuits have become very important due to the high demand for wireless sensor networks and medical applications. Traditional supply voltage scaling has proven to be a valuable technique to significantly reduce energy consumption. Decreasing the supply voltage below the transistor threshold voltage can provide further energy savings. However, sub-threshold operation is often limited to very low performance and suffers severely from the ever increasing variability. Until now, the focus of sub-threshold design was mainly on minimizing energy, resulting in kHz-performance [1]. Two issues must be tackled to allow the widespread use of circuits operating in the sub-threshold region. First, the performance of circuits operating at a low supply voltage in the order of a single $V_{\mathrm{t}}$ must be enhanced from $\mathrm{kHz}$ to $\mathrm{MHz}$ range. Second, variation-resilient circuits must be designed to improve robustness without increasing $V_{\mathrm{dd}}$ despite the exponential sensitivity to variations of sub-threshold circuits.

To eliminate these problems, this paper proposes the use of transmission gate logic in sub-threshold combined with latch-based pipelining which improves performance, enhances variation-resilience and reduces energy consumption. Further, the transmission gate logic is extended with nMOS stacking to reduce leakage and to improve the $I_{\mathrm{on}} / I_{\mathrm{off}}$-ratio. Moreover, the latch-based pipeline enables time borrowing and therefore provides a means to counter the propagation delay variation in sub-threshold circuits.

To prove the proposed techniques, the design of a 32 bit adder which achieves sub-pJ energy consumption combined with MHz-performance is proposed. Section II discusses the optimal gate topology for circuits operating in the weak inversion region. Section III presents the implementation of the
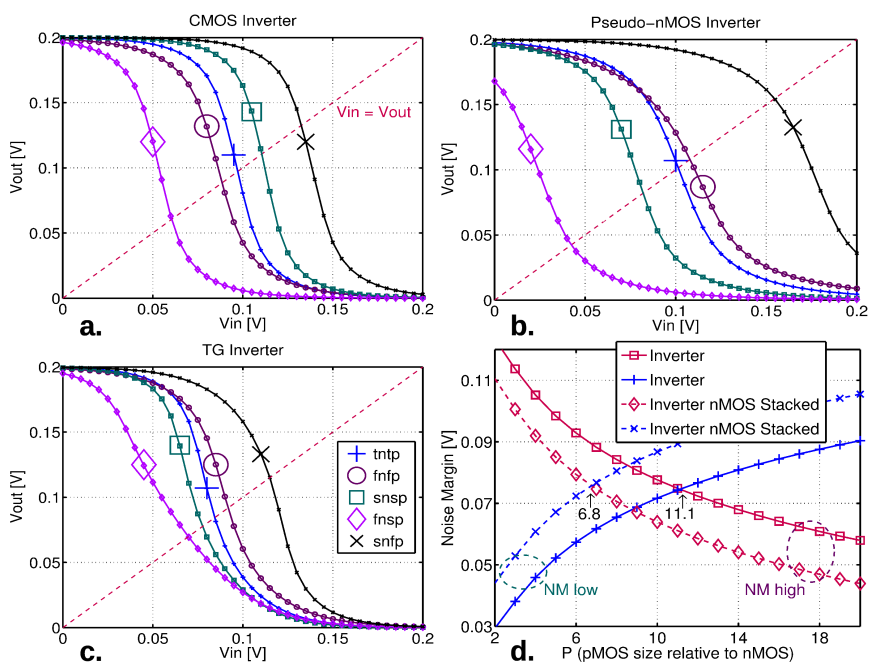

Fig. 1. VTCs $(\mathrm{a}, \mathrm{b}, \mathrm{c})$ of optimally sized inverter topologies for $V_{\mathrm{dd}}=$ $200 \mathrm{mV}$. Process corners are typical, fast and slow, respectively for nMOS and pMOS. Noise Margins (d) of CMOS inverter for $V_{\mathrm{dd}}=200 \mathrm{mV}$.

latch. Section IV describes the proposed design of the adder. Finally, the measurement results are described in section V.

\section{Gate Topology}

The topology used for logic gates is of vital importance for the reliability and performance of sub-threshold circuits. Equal noise margins for high and low levels allow for operation at the lowest supply voltage. For standard CMOS logic gates operating in the sub-threshold region, this can be achieved by sizing or body biasing. The latter technique compensates for inter-die variability by adjusting $V_{\mathrm{t}}$, but requires additional power supply rails and a triple well technology which results in area and energy overhead. With the first technique, i.e. appropriate sizing, a CMOS logic gate also performs well in process corners. Fig. 1a shows the Voltage Transfer Characteristic (VTC) of an optimally sized CMOS inverter for the different process corners. However, for the given technology the best noise margin is obtained with a pMOS/nMOS width ratio of 11 at $V_{\mathrm{dd}}=200 \mathrm{mV}$ (Fig. 1d). Consequently, stacked pMOS devices in e.g. NOR (Fig. 2a) gates require excessive sizes which leads to large area and capacitance and thus deteriorates 


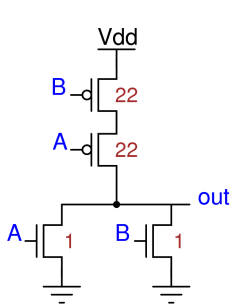

a. CMOS

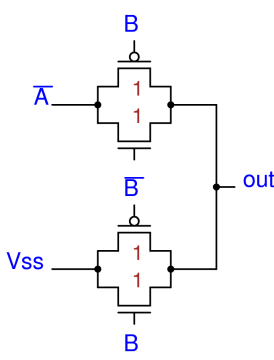

b. TG

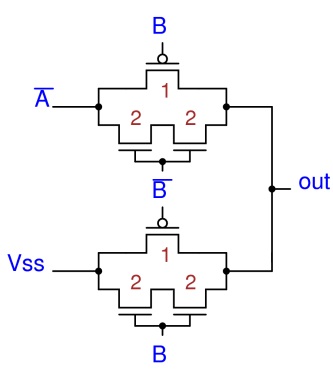

c. TG stacked nMOS
Fig. 2. Schematics of the different NOR topologies.

TABLE I

LEAKAGE POWER AND ENERGY CONSUMPTION COMPARISON FOR DIFFERENT NOR TOPOLOGIES $\left(V_{\mathrm{dd}}=200 \mathrm{mV}\right)$.

\begin{tabular}{l|c|c}
\hline & CMOS & TG stacked nMOS \\
\hline Leakage Power & $3.13 \mathrm{nW}$ & $0.12 \mathrm{pW}$ \\
Dynamic Energy & $64.59 \mathrm{aJ}$ & $6.15 \mathrm{aJ}$ \\
Total Energy @10 MHz & $377.07 \mathrm{aJ}$ & $6.16 \mathrm{aJ}$ \\
\hline
\end{tabular}

the operating speed and energy consumption. Therefore, an alternative gate topology is needed for sub-threshold designs.

Another gate topology previously used for sub-threshold design was Pseudo-nMOS logic [2]. However, these type of ratioed circuits suffer severely from variability, as illustrated by the VTCs of the Pseudo-nMOS in Fig. 1b. This makes Pseudo-nMOS logic undesirable in the sub-threshold region.

A third possibility, transmission gate (TG) logic, has several attractive properties. When a path should conduct current, there are always both an nMOS and a pMOS in parallel that can compensate each other's weaknesses. As Fig. 1c shows, transmission gates suffer less from process variability issues and thus prove to be variation-resilient. Fig. $2 \mathrm{~b}$ shows a NOR gate implemented in transmission gate logic. The pMOS transistors are minimally sized. In this technology, the nMOS $I_{\mathrm{off}, \mathrm{n}}$ is only 21 times lower than the pMOS $I_{\mathrm{on}, \mathrm{p}}$ at $V_{\mathrm{dd}}=200 \mathrm{mV}$. Extensive Monte Carlo simulations of the $I_{\mathrm{on}, \mathrm{p}} / I_{\mathrm{off}, \mathrm{n}}$-ratio resulted in a CDF of which the critical tail is shown in Fig. 3. Nominally the current ratio is already very low, but taking into account $6 \sigma$ intra-die variations the worst-case ratio becomes insufficient, as can be seen from the fitted lognormal distribution on the lower end tail of the ratio with minimal nMOS. An important point to make is that the $I_{\text {on, } \mathrm{p}} / I_{\text {off, }, \mathrm{n}}$-ratio is much smaller than the $I_{\mathrm{on}, \mathrm{n}} / I_{\text {off, } \mathrm{p}}$-ratio. This variability issue is resolved by using the stack effect for the nMOS transistors (Fig. 2c). Stacking two nMOS transistors reduces the nominal $I_{\text {off,n }}$ with a factor of 5 . Therefore, the nMOS stack effect increases the $I_{\mathrm{on}, \mathrm{p}} / I_{\mathrm{off}, \mathrm{n}}$-ratio while the complementary current ratio remains sufficiently high. Even with variations the current ratio with stacked nMOS remains significantly better (Fig. 3). The nMOS stacking introduces a slight speed degradation, but this is outweighed by the reduced leakage and thus the increased robustness. As a result, the sizing of transmission gate logic is relaxed considerably compared to CMOS logic, e.g. the CMOS NOR gate is almost

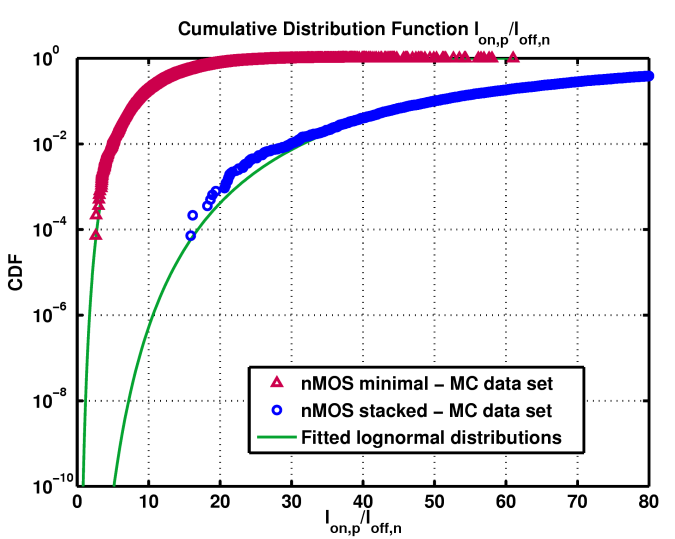

Fig. 3. Critical tail of the Cumulative Distribution Function (CDF) of the $I_{\mathrm{on}, \mathrm{p}} / I_{\mathrm{off}, \mathrm{n}}$-ratio obtained with Monte Carlo simulations and fitted with lognormal distributions. Using stacked nMOS transistors results in a significantly higher worst-case current ratio than using minimal nMOS.

$5 x$ bigger than the TG NOR.

Another important comparison is based on the energy consumption of both topologies (Table I). The TG NOR has the attractive property of an almost non-existing leakage power. This makes the contribution of leakage to the total energy as good as negligible, while the leakage of the CMOS gate has a contribution of $83 \%$ to the total energy consumption. Moreover, the excessive sizing of the CMOS NOR results in a dynamic energy figure that is more than 10x higher than the TG. Hence, the total energy of TG is more than 60x smaller.

To conclude, TG logic is more variation-resilient than CMOS and it consumes significantly less leakage power and dynamic energy. Transmission gate design also avoids pMOS stacking and does not require body biasing. This makes transmission gate logic the most robust option for sub-threshold operation. Therefore, all logic gates used in this design have TG topology. Transmission gates require differential input signals, but the pipelined adder architecture provides these signals in an efficient way (section IV).

\section{LATCH DESIGN}

Fig. 4a shows the schematic of the latch. The latch has a single-input, differential-output functionality to provide differential signals for the TG logic. Other advantages of using latches include enabling pipelining and time borrowing and restoring signal levels. The latch consists of a regular inverter and 2 tristate inverters, controlled by a differential enable signal. The implementation of the regular inverter is shown in Fig. 4b. The inverter is implemented as a CMOS inverter with nMOS stacking. Reducing the nMOS drive strength by stacking relaxes the pMOS sizing for equal noise margins from 11 to 6.8 (Fig. 1d) without a significant speed impact.

Fig. 5 shows two possible implementations of a tristate inverter. On the left, a full-CMOS tristate inverter is given. The enable transistors are placed in the pull-up and pull-down path of the inverter. However, this introduces pMOS stacking and therefore excessive pMOS sizes to ensure good performance in all process corners. The TG-based tristate inverter on the 


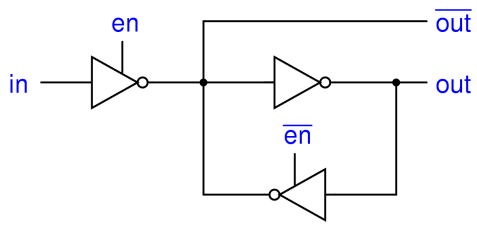

(a) (b)

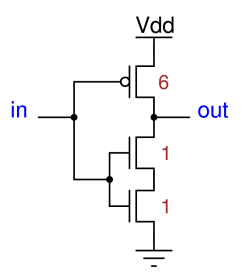

Fig. 4. (a) Schematic of the latch. (b) Transistor implementation of the CMOS inverter with stacked nMOS.

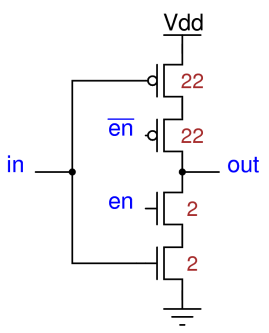

(a)

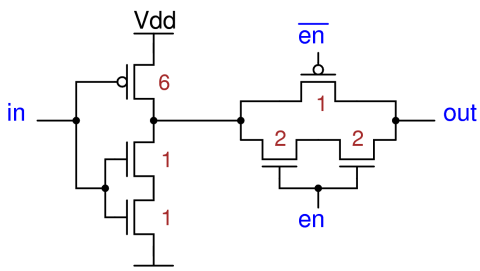

(b)
Fig. 5. Transistor implementations of (a) a full-CMOS tristate inverter and (b) the proposed TG-based tristate inverter which avoids pure pMOS stacking and therefore occupies less area and is more variation-resilient.

right is made transparent by a transmission gate. The pull-up path is therefore extended with nMOS transistors in parallel to the enable pMOS transistor. As a result, pure pMOS stacking is avoided. When considering intra-die variations, the parallel path introduced by the transmission gate allows for compensating the impact of a slower transistor. In addition, because both pull-up and pull-down path include the transmission gate, which consists of both nMOS and pMOS transistors, the sensitivity to process corner variations is reduced. To conclude, the TG-based tristate inverter occupies less area and is more variation-resilient than the full-CMOS tristate inverter.

\section{ADDER DESIGN}

Fig. 6 shows the block diagram of the adder. The topology of the tree adder is Han-Carlson [3], which is a combination of the Brent-Kung (BK) and Kogge-Stone (KS) topologies. BK is characterized by its large number of logic levels, while KS has a high number of wires. Han-Carlson makes a trade-off between these two characteristics and therefore fits best for the TG logic topology. The first and last stage of the Han-Carlson adder are BK, the intermediate stages are KS.

To ensure a high sub-threshold performance, a pipelined architecture is employed to increase the throughput. Pipelining also increases latency, but section $\mathrm{V}$ shows that the latency of this design is still much lower than the comparison point. Variability in sub-threshold circuits results in highly variable gate delays, which makes a pipeline that enables time borrowing [4] preferable in the sub-threshold region. Therefore, this design uses a pipeline based on level-sensitive latches instead of on edge-triggered flip-flops.

Latches are inserted between all stages (Fig. 6). They pro-

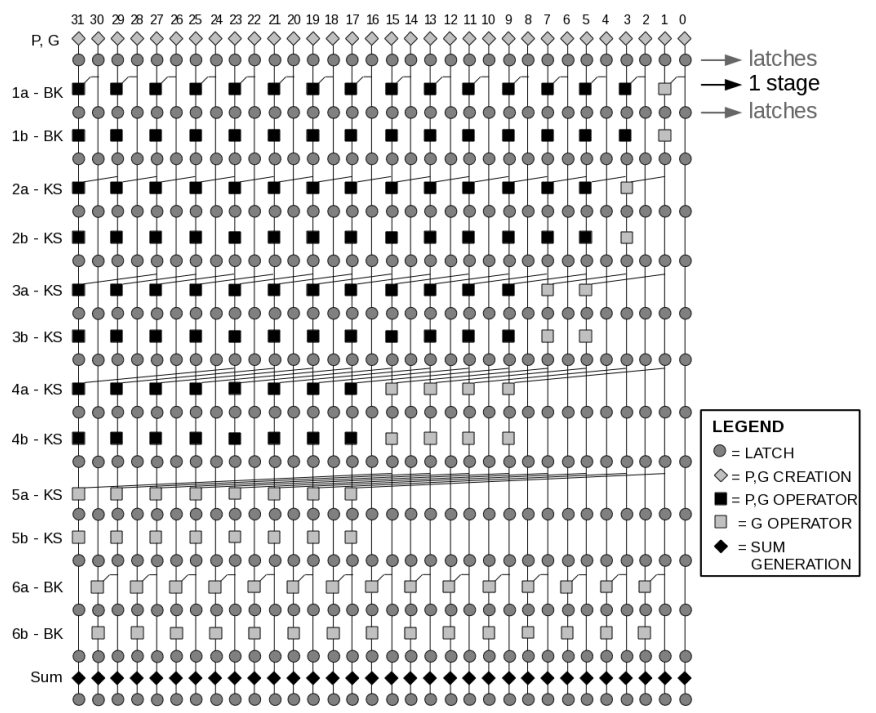

Fig. 6. Block diagram of the 32 bit Han-Carlson adder.

vide differential output signals, which serve as inputs for the transmission gate logic. The $\mathrm{P}, \mathrm{G}$ creation and sum generation stages require 1 logic gate. However, each $\mathrm{P}, \mathrm{G}$ and $\mathrm{G}$ operator stage of the adder requires 2 consecutive logic gates. To ensure minimal and balanced stage delay, those operator stages are split in 2, e.g. the first Brent-Kung stage is split in stages $1 a$ and $1 b$. Thus, every stage consists of 1 TG logic gate. The latch-based pipeline is controlled by non-overlapping clock signals to avoid race conditions.

\section{Measurements}

Measurement results of energy consumption and clock frequency of the adder as function of $V_{\mathrm{dd}}$ are given in Fig. 7 . Operational frequencies in the 10 to $100 \mathrm{MHz}$ range are achieved at an energy consumption per addition that is below $1 \mathrm{pJ}$. The adder operates down to a supply of $190 \mathrm{mV}$ where a clock frequency of $10 \mathrm{MHz}$ and an energy consumption of $0.4 \mathrm{pJ}$ are obtained. A performance of $30 \mathrm{MHz}$ is obtained at a $260 \mathrm{mV}$ supply and $0.6 \mathrm{pJ}$ per addition. The energy increases approximately linearly with $V_{\mathrm{dd}}$, while the clock frequency increases roughly quadratically (Fig. 7). An application requiring higher performance can thus achieve this at a relatively low energy cost, which demonstrates the versatility of this design. Table II depicts the measurement results and the Energy-Delay Product (EDP), which is the Figure Of Merit that balances the importance of both energy and performance.

Fig. 8 shows the measured contribution of the leakage power to the total power consumption of the 32 bit adder as function of $V_{\mathrm{dd}}$. The total power consumption is measured at the maximum operating frequency for every supply voltage. The leakage power is measured when the adder is idle. At a supply of $190 \mathrm{mV}$, the leakage power is $1.65 \mu \mathrm{W}$ and leakage consumes $42.6 \%$ of the total power consumption. As $V_{\mathrm{dd}}$ lowers and the circuit operates more in the sub-threshold region, leakage power becomes an important part of overall power consumption. To overcome the idle leakage problem, an 


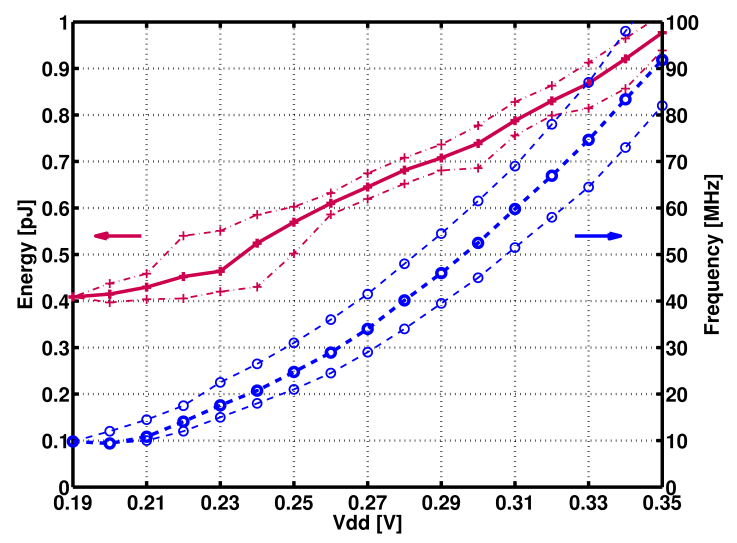

Fig. 7. Measured energy and clock frequency as function of $V_{\mathrm{dd}}$. Average values are in bold, surrounded by the maximum and minimum measured values out of 8 dies. The values mentioned in the text are average values.

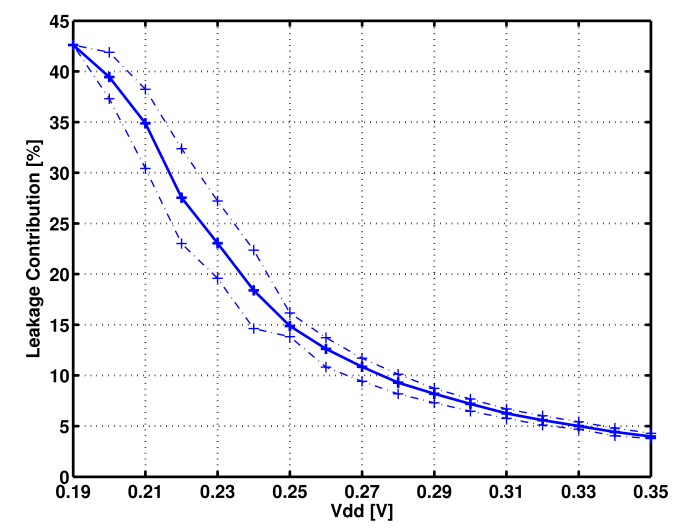

Fig. 8. Measured contribution of the leakage power to the total power consumption as function of $V_{\mathrm{dd}}$ during operation. In standby mode, power gating is applied which results in an idle leakage reduction of a factor 10.

on-chip power gating scheme is employed to reduce leakage in standby mode. When the adder is idle, the power supply and ground are cut off by 2 power switches, resulting in a measured standby leakage reduction of approximately a factor 10 .

Table II compares this adder with the only other published $90 \mathrm{~nm}$ CMOS, 32 bit sub-threshold logarithmic adder [1]. Other recent work on digital sub-threshold designs focusses on processors (e.g. [5], [6]) rather than variation-resilient and ultra-low-energy datapath elements. Since this would not yield a meaningful comparison, these papers are not included in Table II. This work outperforms the Kogge-Stone adder in both throughput and latency. While [1] operates in the kHz-range in the sub-threshold region, this Han-Carlson adder functions in the MHz-range. The referenced adder focussed on minimum energy operation and obtains a lower energy consumption, while the new design achieves both ultra-low-energy and $\mathrm{MHz}$-performance, as is obvious from the improvement of the EDP, which is 160 to 900 times lower than the EDP of [1].

Layout placement and routing is performed with the dedicated Datapath Generator [7] in order to obtain a dense layout while drastically reducing layout effort compared to full-custom layout. Fig. 9 shows the chip micrograph of the adder. The active area of the adder is $650 \times 50 \mu \mathrm{m}^{2}$.
TABLE II

STATE-OF-THE-ART SUB-THRESHOLD ADDER COMPARISON.

\begin{tabular}{|c|c|c|c|c|c|c|c|c|c|}
\hline & \multicolumn{2}{|c|}{ [1]: } & \multicolumn{2}{|c|}{ Kogge-Stone } & \multicolumn{2}{|c|}{ This Work: } & \multicolumn{2}{|c|}{ Han-Carlson } & \multirow[b]{2}{*}{$\begin{array}{c}\text { EDP } \\
\text { Factor }\end{array}$} \\
\hline $\begin{array}{l}V_{\mathrm{dd}} \\
{[\mathrm{mV}]}\end{array}$ & $\begin{array}{c}\mathrm{T} \\
{[\mathrm{Hz}]}\end{array}$ & & $\begin{array}{c}\text { Energy } \\
{[\mathrm{pJ}]}\end{array}$ & $\begin{array}{c}\text { EDP } \\
{[\mathrm{pJ} . \mu \mathrm{s}]}\end{array}$ & $\begin{array}{c}\mathrm{T} \\
{[\mathrm{Hz}]}\end{array}$ & $\begin{array}{c}\mathrm{L} \\
{[\mu \mathrm{s}]}\end{array}$ & $\begin{array}{c}\text { Energy } \\
{[\mathrm{pJ}]}\end{array}$ & $\begin{array}{c}\text { EDP } \\
{[\mathrm{pJ} . \mu \mathrm{s}]}\end{array}$ & \\
\hline 190 & - & - & - & - & $9.5 \mathrm{M}$ & 1.47 & 0.408 & 0.0 & - \\
\hline 250 & $7 \mathrm{k}$ & 143 & 0.145 & 20.7 & $24.8 \mathrm{M}$ & I 0.57 & 0.569 & 0.023 & 900 \\
\hline 300 & $25 \mathrm{k}$ & 40 & 0.10 & 4.0 & $52.5 \mathrm{M}$ & I 0.27 & 0.739 & 0.0 & 285 \\
\hline 330 & $50 \mathrm{k}$ & 20 & 0.095 & 1.9 & $74.6 \mathrm{M}$ & I 0.19 & 0.868 & 0.012 & 160 \\
\hline
\end{tabular}

$\mathrm{T}=$ Throughput, $\mathrm{L}=$ Latency

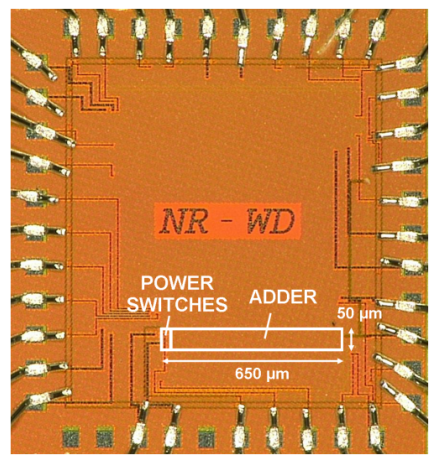

Fig. 9. Chip micrograph of the 32 bit adder.

\section{CONCLUSION}

This work has presented a sub-threshold 32 bit adder with a latch-based pipelined architecture. Sub-threshold adapted transmission gate logic, optimal sizing for noise margins and time borrowing are used as variation-resilient circuit techniques. This sub-threshold design targets MHz-performance at an ultra-low energy consumption per addition. At a minimal supply of $190 \mathrm{mV}$, the energy consumption is $0.4 \mathrm{pJ}$ at a $10 \mathrm{MHz}$ clock frequency. This work achieves a significant improvement in Energy-Delay Product of up to 900 compared to the state-of-the-art in sub-threshold adder design. Power gating is applied to reduce standby leakage power.

\section{ACKNOWLEDGMENT}

This work is supported by the Research Foundation Flanders (FWO). The authors would like to thank Tobias Noll and Oliver Weiss from RWTH Aachen for the opportunity to work with Datapath Generator and the provided support.

\section{REFERENCES}

[1] B. Calhoun and A. Chandrakasan, "Ultra-dynamic voltage scaling using sub-threshold operation and local voltage dithering," JSSC, pp. 238-245, Jan. 2006.

[2] H. Soeleman and K. Roy, "Ultra-low power digital subthreshold logic circuits," in ISLPED, 1999, pp. 94-96.

[3] T. Han and D. Carlson, "Fast area-efficient VLSI adders," in IEEE Symp. Computer Arithmetic, May 1987, pp. 49-56.

[4] D. Harris, Skew-Tolerant Circuit Design. Morgan Kaufmann Publ., 2001.

[5] Y. Pu et al., "An ultra-low-energy multi-standard JPEG co-processor in 65nm CMOS with sub/near threshold supply voltage," JSSC, pp. 668680, Mar. 2010.

[6] S. Hanson et al., "Exploring variability and performance in a sub-200-mv processor," JSSC, pp. 881-891, Apr. 2008.

[7] O. Weiss, M. Gansen, and T. Noll, "A flexible datapath generator for physical oriented design," in ESSCIRC, Sep. 2001, pp. 393-396. 